\title{
CLOUD BASED PROJECT MANAGEMENT IN THE GREEK CONSTRUCTION INDUSTRY: WHAT IS THE DISTANCE TO COVER?
}

\author{
Yiannis Xenidis ${ }^{1}$, Emmanouil Chiotakis ${ }^{2}$
}

\begin{abstract}
Cloud computing promises to revolutionize traditional construction project management through reduced costs and access to a project's central information platform from any device connected to the internet. Cloud-Based Project Management (CBPM) is designed to automate and standardize the various processes in the management of Engineering and Construction $(\mathrm{E} \& \mathrm{C})$ projects by providing a cooperation platform for all project stakeholders, which allows real time updating of important information as well as reduction of time frictions in communication. This research is the first structured approach to investigate: a) the degree of understanding of CBPM from the Greek Construction Industry (GCI), b) the degree of incorporation of CBPM in GCI and c) the potential for further addressing CBPM in GCI, as well as the needed changes to the industry to achieve this incorporation. Research methodology comprised two stages: a) a literature review, which identified the benefits from utilizing specific CBPM tools and the necessary actions for integrating them in $\mathrm{E} \& \mathrm{C}$ companies, and b) an interview of 38 professionals that revealed challenges and opportunities of $\mathrm{E} \& \mathrm{C}$ organizations in the GCI with regard to the implementation of CBPM. The research's findings are indicative for the near future of CBPM in the Greek Construction Industry.
\end{abstract}

Keywords: Cloud Computing, Cloud-Based Project Management, Construction Management, Construction Industry, Building Information Modeling

\section{INTRODUCTION}

The implementation of Engineering and Construction (E\&C) projects with conventional project management processes is often characterized by crunches and problems in the design and construction phases. Some of the most frequent ones are (Braglia and Frosolini 2014; Chong et al. 2014):

- E\&C projects often fall behind schedule

- $\mathrm{E} \& \mathrm{C}$ projects' timetables are often redesigned due to limitations of financial resources or inefficient incorporation of specifications and requirements to the original design.

- $\mathrm{E} \& \mathrm{C}$ projects often suffer from cost overruns, thus leading to excessive funding requirements.

Assistant Professor, School of Engineering, Department of Civil Engineering, Director of the Construction Equipment and Management Laboratory, Aristotle University of Thessaloniki, Thessaloniki, GR, ioxen@civil.auth.gr

2 Graduate, School of Engineering, Department of Civil Engineering, Aristotle University of Thessaloniki,echiotak@civil.auth.gr 
- $\mathrm{E} \& \mathrm{C}$ projects may be very complicated leading to inefficient tasks performance due to lack of clarity in the assignments (e.g. who's doing what and for how long) or coinciding tasks, etc.

- E\&C projects often suffer from inefficient supply chains, thus leading to delays in tasks execution and excessive need for re-planning during the projects' development

- E\&C projects often suffer from inefficient communication protocols between workers, engineers and managers, thus leading to mismanagement, conflicts, demoralization in the working environment, etc.

The multidimensional nature of $\mathrm{E} \& \mathrm{C}$ projects performance and the large number of factors that affect it in an, often, unpredictable way, clearly give prominence to the need for excellent cooperation between the projects' various stakeholders. Modern project management has undertaken those issues through technologically advanced software tools; however, until recently, local based project management software strived to address the problem of different cooperation protocols between the stakeholders in $\mathrm{E} \& \mathrm{C}$ projects (Marston et al. 2011). Moreover, this kind of software operates inside office boundaries, thus resulting to reduced interoperability between the project management tools used by the various stakeholders at the different stages of the project's implementation (Moses et al. 2008). This shortage leads in turn to the inefficient transfer of project's significant information due to the lack of standardized operation protocols (Watson, 2011).

The new emerging generation of project management tools addresses specialized and modernized software that is based on the "Cloud Computing" ecosystem standards. "Cloud Computing" offers software applications to end users by instantly transmitting information via the Internet and dynamically allocating computing resources, on demand, through a smart task management system. (Curry et al. 2013; Yi et al. 2013; Chong et al. 2014). The "Cloud Computing" ecosystem is based on the wider concept of "Converged Infrastructure" or else, software structures that converge and create a unified Information Technology (IT) operating platform through shared services (Curry et al 2013). At the same time, data migration into the "cloud" is an emerging trend, which is based on the idea that each requested information on a particular topic should be provided to interested parties at any requested time by cloud software providers (Curry et al. 2013; Chang et al. 2013). As a result, individual users have the ability to use "Cloud Computing" services provided via the Internet, without any restriction (geographical, organizational, etc.) except of those imposed in the project's managerial context (Curry et al 2013).

While the entire above is applied in the same identical way to the construction industry worldwide, there are various and often different problems that local construction industries face with a significant impact on their capacity to catch up with technology developments and achieve the culture transformation required to remain competitive in the global environment. Especially in Greece, the construction industry was severely hit by the economic financial crisis in 2009 and the continuous recession till now by losing $80 \%$ of its output only in the years 2010-2013 (Building Radar GmbH, 2016). This situation has prevented, until now, the former growth engine of the national economy to follow the growth rates that the construction industry largely presents throughout Europe since 2014. Moreover, while the inner structure of the European construction industry has remained intact, in Greece all relevant aspects (e.g., employment, number of companies) have been dramatically reduced (Building Radar 
$\mathrm{GmbH}, 2016)$. In such a context, it is important to determine the level of readiness of the construction industry to adapt and apply high-end technological solutions, in order to identify the necessary steps towards regaining competitiveness at the global level.

This research is the first systematic effort to identify: a) the degree of understanding of Cloud Based Project Management (CBPM) software from the Greek Construction Industry and b) the degree of incorporation of CBPM tools to GCI. The applied research tool was in person interviews with 21 employees from $\mathrm{E} \& \mathrm{C}$ organizations specialized in public as well as private construction projects (15 and six employees, respectively). The sample included also employees from 17 municipality agencies that are responsible for supervising construction projects, achieving in this way a comprehensive and in depth view of CBPM software awareness from and incorporation in the GCI. The interview that was semi structured and involved 19 questions was evaluated as the most effective research tool compared to others (e.g. questionnaires) considering its increased performance in dealing with the following constraints placed by the Greek industry's low level of familiarity with the topic: a) small sample available to participate to the research, b) demand for interaction between the researchers and the respondents for clarifying the questions' content when necessary, and c) unknown level of quality and accuracy of the collected responses (Naoum 2007).

The structure of this paper is the following: a) Section 2 presents the awareness of general cloud based software in GCI, b) Section 3 presents the degree of CBPM and especially cloud based "BIM" software adoption in GCI, c) Section 4 presents the challenges incorporating CBPM tools in the GCI, and d) Section 5 concludes with the survey's overall results.

\section{GREEK CONSTRUCTION INDUSTRY'S AWARENESS OF GENERAL ClOUd BASED SOFTWARE}

The starting point of this research is the identification of the awareness that the Greek Construction Industry has with regard to Cloud Based Software (CBS). Table 1 provides an interesting mapping of the level of awareness of different groups of organizations in CGI.

Table 1: General awareness of cloud based software in GCI.

\begin{tabular}{|c|c|c|c|}
\hline Type of Organization & Great & Minor & None \\
\hline Municipality Agency - MA & $5,9 \%$ & $64,7 \%$ & $29,4 \%$ \\
\hline $\begin{array}{l}\text { E\&C Companies offering services to the } \\
\text { Public Sector - ECPuS }\end{array}$ & $6,7 \%$ & $60,0 \%$ & $33,3 \%$ \\
\hline $\begin{array}{c}\text { E\&C Companies offering services to the } \\
\text { Private Sector - ECPrS }\end{array}$ & $0,0 \%$ & $33,3 \%$ & $66,7 \%$ \\
\hline
\end{tabular}

It is clear that the general awareness of CBS by the GCI is between minor and none. The levels of some awareness (between minor to great) are significantly higher for $\mathrm{E} \& \mathrm{C}$ companies that work with the public sector compared to the other groups, while municipality agencies rank second. It is surprising, though, that private sector construction companies present a very low awareness of CBS, far from what would be 
normally anticipated considering the increased potential for innovation that the private sector has compared to the public sector.

The investigation of the general awareness of CBS was not limited to a single straightforward question (e.g., "Are you familiar with CBS?") but was rather explored through more questions. The aim was to identify the actual use of CBS in these organizations and overcome the users' own impression of the level of acknowledgement they have for the nature of the software they use. In this context, the interviewed people were asked about the level of use of specific services and applications that CBS offers. As Table 2 shows, common cloud services are already used in the GCI. Cloud based documents management seems to be significantly widespread, while remote accessibility has a potential for further grow. Although the respondents are familiar with CBS features that offer mobility, ease of access and easier communication between colleagues (e.g., use of software from any device connected to the Internet, personal work done from any mobile device connected to the Internet, collaboration with other users, etc.) and potential for online collaboration, these are still less developed compared to the use of services listed in Table 2.

Table 2: Applications of CBS in GCI.

\begin{tabular}{cccc}
\hline \multirow{2}{*}{ Type of service } & \multicolumn{3}{c}{ Type of Organization } \\
\cline { 2 - 4 } & MA & ECPuS & ECPrS \\
\hline $\begin{array}{c}\text { Upload documents and online study } \\
\text { of documents }\end{array}$ & $82,4 \%$ & $100,0 \%$ & $100,0 \%$ \\
Share files & & & \\
Synchronize accounts between & $64,7 \%$ & $93,3 \%$ & $50,0 \%$ \\
different devices & $23,5 \%$ & $46,7 \%$ & $0,0 \%$ \\
Use of apps from smart phones & $52,9 \%$ & $66,7 \%$ & $50,0 \%$ \\
\hline
\end{tabular}

The combined results from Table 1 and Table 2 clearly reveal that GCI is actually using cloud based software; however, it is not aware of doing so or of the extent of utilization that could be achieved. Understanding the context of the tools used in everyday practice would allow GCI to further: a) exploit the potential benefits of already used applications, b) investigate more applications of utility to the industry, and c) incorporate in a systematic way more IT applications to the industry.

\section{Cloud Based Project Management in the GreeK CONSTRUCTION INDUSTRY}

CBS can facilitate project management in a much more focused approach than the types of services described in Table 2; however, the transition from Computer-Aided Project Management (CAPM) to Cloud Based Project Management is not an easy task. The literature review reveals several obstacles that prevent professionals in the construction industry from incorporating CBPM in their process, including: a) different levels (even lack) of expertise between stakeholders in using project management software, b) false assumptions for high investment costs, c) reluctance in sharing information via the Internet, etc. (Ku and Taeibat 2011; Mayo et al. 2012; Eadie et al. 2013). 
GCI presents a very low performance in addressing both CAPM and CBPM in standard professional practice, as the respective interview responses reveal in Table 3.

Table 3: Incorporation of CAPM and CBPM in GCI's practice.

\begin{tabular}{cccc}
\hline \multirow{2}{*}{ Investigated issue } & \multicolumn{3}{c}{ Type of Organization } \\
\cline { 2 - 4 } & $\mathrm{MA}$ & $\mathrm{ECPuS}$ & $\mathrm{ECPrS}$ \\
\hline $\begin{array}{c}\text { Incorporation of any software with } \\
\text { cloud architecture }\end{array}$ & $0,0 \%$ & $86,7 \%$ & $16,7 \%$ \\
$\quad \begin{array}{c}\text { Use of specific PM software } \\
\text { Combined use of PM software and }\end{array}$ & $0,0 \%$ & $46,7 \%$ & $0,0 \%$ \\
$\quad$ CAD solutions & $0,0 \%$ & $0,0 \%$ & $0,0 \%$ \\
\hline
\end{tabular}

As shown in Table 3, municipality agencies are completely detached from a CAPM and CBPM approach, while E\&C companies offering services to the private sector are barely using some cloud-based applications. While E\&C companies offering services to the public sector seem to be in a better position with regard to the incorporation of CBPM, none of the three types of organizations uses Project Management (PM) software in combination with CAD solutions. It is necessary to clarify that the investigated issues in Table 3 refer to typical (standard) organizational (professional) practice and not on individual practices that may be applied by the employees in these organizations. In other words, the interviews reveal that there is no typical authorization of CAPM and CBPM solutions in standard practice; however, based on the findings presented in Table 2 , it can be, safely, assumed that this is not a result of ignorance of these solutions, but rather the outcome of the combination of the usual obstacles that were mentioned above.

The limited application of CAPM naturally leads to a scarce knowledge of Building Information Modeling (BIM) solutions in GCI, as shown in Table 4.

Table 4: GCI's awareness of Building Information Modeling (BIM) solutions.

\begin{tabular}{cccc}
\hline Type of Organization & Great & Minor & None \\
\hline $\begin{array}{c}\text { Municipality Agency - MA } \\
\text { E\&C Companies offering services to the } \\
\quad \text { Public Sector - ECPuS }\end{array}$ & $0,0 \%$ & $5,9 \%$ & $94,1 \%$ \\
$\begin{array}{c}\text { E\&C Companies offering services to the } \\
\text { Private Sector - ECPrS }\end{array}$ & $0,0 \%$ & $0,0 \%$ & $100,0 \%$ \\
\hline
\end{tabular}

The limited application of CAPM and CBPM and the very limited awareness of BIM that is recorded in Table 4 collectively demonstrate the poor GCI's performance with regard to the incorporation of IT solutions in $\mathrm{E} \& \mathrm{C}$ projects, but also indicate a culture that practically ignores interoperability, a notion which is dominant in CBPM software (Yi et al 2013). No matter how contradictory this is to the widespread use of, especially, CAPM solutions and the plethora of information around IT solutions in $\mathrm{E} \& \mathrm{C}$ projects, it clearly 
shows that GCI needs to move fast towards the acquaintance with critical notions and technologies before applying them in standard practice.

\section{CHALLENGES IN INCORPORATING CBPM SOFTWARE IN THE GREEK CONSTRUCTION INDUSTRY}

The incorporation of CBPM software in any $\mathrm{E} \& \mathrm{C}$ company requires a number of adaptations and changes that extend over the whole organization. Chiotakis (2016) describes the most critical of those adaptations that are related to: a) the existing equipment and hardware infrastructure, b) the compatibility of existing software with CBS, c) the communication protocols with the various project stakeholders, d) the communication protocols and collaboration inside the organization, e) the processing of e-material, including e-mail messages, files, etc., f) the training programs for the staff with regard to the use of CBPM software, g) the project monitoring by the project manager, and h) the organization's culture with regard to the incorporation of new technologies.

Beyond those adaptations, which are more of a technical nature, the interviews in this research highlighted a set of specific challenges, which are listed in Table 5, and are more related to the perceptual and organizational aspects of the incorporation of CBPM software into GCI.

Table 5: Challenges in incorporating CBPM software in GCI.

\begin{tabular}{cc}
\hline Aspect & Challenge \\
\hline Perceptual & $\begin{array}{c}\text { Persuade small and medium E\&C companies for the benefits of CBPM } \\
\text { Reverse the false perception of high costs for applying CBPM } \\
\text { Organizational }\end{array}$ \\
& Exploit the well educated young engineers \\
& Confront change resistance during application of CBPM \\
& Deal with security issues as well as intellectual property rights \\
\hline
\end{tabular}

The challenges listed in Table 5 provide the outline of an industry that is currently unprepared to introduce and make an effective use of the new technologies for managing $\mathrm{E} \& \mathrm{C}$ projects. The literal responses that are concisely presented in Table 5 reveal a conservative approach in $\mathrm{E} \& \mathrm{C}$ project management with a low level of exploitation of IT solutions and reluctance by the senior managers to introduce such solutions. This hesitation is further increased from the unwillingness to invest in new technologies, especially in a period of recession. As a result, while the industry acknowledges the capacity of the younger engineers to successfully operate CBPM software, GCI remains stagnant with regard to the incorporation of this type of software in everyday practice.

\section{CONCLUSIONS}

$\mathrm{E} \& \mathrm{C}$ project management is known to suffer from various problems, which despite their repetitiveness and similarity in appearance remain complex and hard to solve even for the most experienced professionals. A main source of these problems is the lack of interoperability between the various stakeholders involved in these projects. Cloud Based 
Software solutions effectively respond to the need of increasing the stakeholders' interoperability in all aspects of project management, while at the same time they minimize costs and literally transform the project management practice in the construction industry.

This research, for the first time, investigated in a systematic and scientifically sound approach the Greek Construction Industry's awareness of Cloud Based Project Management, the level of CBS application in the industry and the challenges that GCI faces towards the transition to a more IT based operation. Semi structured interviews were designed and conducted with 38 individuals coming from different sectors of the $\mathrm{E} \& \mathrm{C}$ industry, namely municipality agencies, $\mathrm{E} \& \mathrm{C}$ companies undertaking public works, and $\mathrm{E} \& \mathrm{C}$ companies undertaking projects from the private sector.

The first significant finding of this research is GCI's low level of awareness of CBS. More specifically, it is verified that common CBS applications are known and even used at the individual level, while employees are unaware both of the full range of the benefits that these applications may offer as well as of the context of cloud management per se.

Another important finding is that this general awareness of CBS is limited at the individual level and is very far from being considered as organizational knowledge, since there is hardly any typical introduction of CBPM processes in the studied organizations. In fact, the most advanced use of CBS concerns well-known commercial products and apps from smart phones for uploading, sharing and studying (online) documents, while, even this use is limited to $\mathrm{E} \& \mathrm{C}$ companies offering services to the public sector. There is hardly any use of specific PM software and the combined use of PM software with CAD solutions was not recorded at all.

The research, also, revealed a more fundamental problem towards the transformation from conventional project management to CBPM. This is the fact that there is hardly any use of specific PM software in GCI and the combined use of PM software with CAD solutions is almost completely absent. These findings clearly show that GCI is rather detached from a computer aided project management approach, a fact that further hinders the transition to cloud based project management. For example, despite the rapid application of BIM in the construction industry worldwide, GCI has a very limited knowledge about it.

All the above findings clearly suggest that there is a long distance to cover towards the effective use of CBS in GCI. The crucial adaptations that GCI needs to make do not concern the usual technical and technological ones that are anticipated in any similar transition from conventional to IT based operation. They rather concern perceptual and organizational aspects, which are directly affected by the industry's culture. The latter is rather conservative and characterized by strong, yet unreasoned beliefs that an investment on CBS is unnecessary and non-profitable and has small benefits to offer in project management, especially for small and medium E\&C companies. These unjustified and arbitrary opinions oppose changes in the Greek construction industry and hinder the effective utilization of the new generations of engineers that are much more familiar to IT solutions and possess the capacity, knowledge background and quality to successfully apply these solutions to the industry's common practice. Therefore, the biggest challenge that GCI faces towards the transition from conventional project management to CBPM is changing the culture and investing on new technologies that have the potential to increase productivity, efficiency and effectiveness of E\&C projects in Greece. 


\section{REFERENCES}

Braglia, M. and Frosolini, M. (2014). An Integrated Approach to Implement Project Management Information Systems within the Extended Enterprise. International Journal of Project Management, 32(1), pp. 18-29, doi: 10.1016/j.ijproman.2012.12.003.

Building Radar GmbH (2016). Construction Industry in Europe. Available at: https://buildingradar.com/construction-blog/construction-industry-europe/ [Accessed: 30 Mar. 2017]

Chang, V., Walters, R.J. and Wills, G. (2013). The Development that Leads to the Cloud Computing Business Framework. International Journal of Information Management, 33(3), pp. 524- 538, doi: 10.1016/j.ijinfomgt.2013.01.005.

Chiotakis, E. (2016). Cloud Project Management in Construction Projects. MSc Thesis, Department of Civil Engineering, Aristotle University of Thessaloniki.

Chong, H.-Y., John, S. W. and Wang, X. (2014). An Explanatory Case Study on Cloud Computing Applications in the Cloud Computing Applications in the Built Environment. Automation in Construction, 44, pp. 152-162, doi: 10.1016/j.autcon.2014.04.010.

Curry, E., O’Donnell, J. and Corry, E. (2013). Linking Building Data in the Cloud: Integrating Cross-domain Building Data Using Linked Data. Advanced Engineering Informatics, 27(2), pp. 206-219, doi: 10.1016/j.aei.2012.10.003.

Eadie, R., Browne, M. Odeyinka, H., McKeown, C., and McNiff, S. (2013). BIM Implementation throughout the UK Construction Project Lifecycle: An Analysis. Automation in Construction, 36, pp. 145-151, doi: 10.1016/j.autcon.2013.09.001.

Ku, K. and Taeibat, M. (2011). BIM Experiences and Expectations: The Constructors' Perspective. International Journal of Construction Education Research, 7(3), pp.175197, doi: 10.1080/15578771.2010.544155.

Marston, S., Bandyopadhyay, S., Zhang, J. and Ghalsasi, A. (2011). Cloud Computing The Business Perspective. Decision Support Systems, 51(1), pp. 176-189, doi: 10.1016/j.dss.2010.12.006.

Mayo, G., Giel, B. and Issa, R.R.A. (2012). BIM Use and Requirements among Building Owners. In: Issa, R.R. and Flood, I. (eds.), International Conference on Computing in Civil Engineering, Clearwater Beach, Florida, USA, 17-20 June 2012. Reston, Virginia: ASCE.

Moses, S., El-Hamalawi, A. and Hassan, T. (2008). The Practicalities of Transferring Data between Project Collaboration Systems used by the Construction Industry. Automation in Construction, 17(7), pp. 824-830, doi: 10.1016/j.autcon.2008.02.007.

Naoum, S.G. (2007). Dissertation Research and Writing for Construction Students. Elsevier Ltd., Oxford, UK.

Watson, A. (2011). Digital Buildings - Challenges and Opportunities. Advanced Engineering Informatics, 25(4), pp. 573-581, doi:10.1016/j.aei.2011.07.003.

Yi, J., Yinghui, W., Shaohua, Z., Yin, L., Baoming, Y. and Lei, Y. (2013). A Cloud Approach to Unified Lifecycle Data Management in Architecture, Engineering, Construction and Facilities Management: Integrating BIMs and SNS. Advanced Engineering Informatics, 27(2), pp. 173-188, doi: 10.1016/j.aei.2012.11.006. 